\title{
TI.11.1
}

\section{A Recipe for Configuring and Operating LDAP Directories (2.1)}

- PDF: 00010-ARecipeForConfiguringAndOperatingLDAPDirectories.pdf

- HTML: Idap-recipe.htm

\section{More Information}

\begin{tabular}{|l|l|}
\hline Repository ID & TI.11.1 \\
\hline Persistent URL & http://doi.org/10.26869/TI.11.1 \\
\hline Title & A Recipe for Configuring and Operating LDAP Directories (2.1) \\
\hline Authors & Michael R Gettes \\
\hline Sponsor & MACE \\
\hline Review & \\
\hline Status & Legacy \\
\hline Publish Date & October 10,2002 \\
\hline DOI & $10.26869 /$ TI.11.1 \\
\hline Signature & \\
\hline Deprecated & No \\
\hline Future Review & \\
\hline Supersedes & \\
\hline Format & PDF \\
\hline Related Docs & \\
\hline Development Location & \\
\hline IP Framework & \\
\hline Subject Tags & middlewarerescue \\
\hline Notes & \\
\hline
\end{tabular}

